\title{
Molecular and pathological characterization of the EZH2 rs3757441 single nucleotide polymorphism in colorectal cancer
}

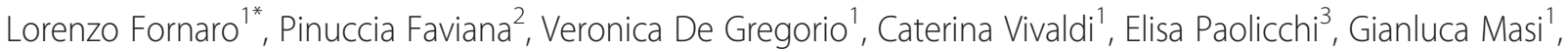 \\ Fotios Loupakis ${ }^{1}$, Elisa Sensi ${ }^{2}$, Cristiana Lupi ${ }^{2}$, Gabriella Fontanini ${ }^{2}$, Yuzhuo Wang ${ }^{4,5}$, Romano Danesi ${ }^{6}$, \\ Alfredo Falcone ${ }^{1}$ and Francesco Crea ${ }^{4,7^{*}}$
}

\begin{abstract}
Background: The enhancer of zeste-homolog $2(E Z H 2)$ is involved in cancer development through gene silencing by trimethylation of lysine 27 of histone 3 (H3K27me3). The C/C genotype for the EZH2 rs3757441 single-nucleotide polymorphism (SNP) is linked with poor prognosis in metastatic colorectal cancer (CRC), but molecular and pathological characterization of this SNP is lacking.

Methods: 119 primary CRCs were analyzed. SNP was evaluated by real-time PCR from colonic healthy tissue, while EZH2 and H3K27me3 expression were studied by immunohistochemistry. We primarily looked for correlation between EZH2 rs3757441 genotypes and EZH2/H3K27me3 expression. Potential associations between EZH2/H3K27me3 expression and clinico-pathological features or KRAS exon 2 and BRAF exon 15 mutations were secondary endpoints. Statistical analysis was performed by chi-square test, T-test or ANOVA.

Results: The C/C genotype was significantly associated with higher EZH2 (100 vs. 44\%; $P=0.019)$ and H3K27me3 (100 vs. $38 \% ; P=0.009$ ) staining intensity compared with $C / T$ and $\mathrm{T} / \mathrm{T}$. EZH2 3+ staining significantly correlated with stronger H3K27me3 expression $(P=0.039)$. KRAS and BRAF mutations were not associated with $\mathrm{EZH} 2$ or H3K27me3 expression.
\end{abstract}

Conclusion: $E Z H 2$ rs3757441 C/C genotype is associated with stronger EZH2 and H3K27me3 immunoreactivity in primary CRC: this SNP may serve as a promising biomarker for EZH2-targeting agents and may add independent information to KRAS and BRAF testing.

Keywords: BRAF, Colorectal cancer, EZH2, KRAS, Polycomb, Single nucleotide polymorphism

\section{Background}

Colorectal cancer $(\mathrm{CRC})$ is one of the leading causes of cancer-related deaths worldwide [1]. Validated predictive or prognostic biomarkers in metastatic CRC (mCRC) are limited to RAS and BRAF mutations [2,3]. There is therefore a dire need for a deeper characterization of CRC biology and for the identification of innovative therapeutic targets.

\footnotetext{
*Correspondence: lorenzo.fornaro@gmail.com; francesco.crea@open.ac.uk 'Unit of Medical Oncology 2, Azienda Ospedaliero-Universitaria Pisana, Istituto Toscano Tumori, Pisa, Italy

${ }^{4}$ Experimental Therapeutics, BCCA Cancer Research Centre, Vancouver, BC, Canada

Full list of author information is available at the end of the article
}

Epigenetics offers a different perspective to understand cancer biology, complementing the conventional genetic approach [4]. Polycomb Group genes (PcGs) represent some of the most studied components of the epigenetic machinery. PcGs are organized in two major Repressive Complexes (PRCs) and induce gene silencing through histone post-translational modifications [5]. Most PcGs have been involved in the initiation and promotion of cancer and contribute to drug resistance in both haematological and solid malignancies [6].

$\mathrm{EZH} 2$ is the catalytic subunit of PRC2, which catalyzes histone H3 trimethylation on lysine 27 (H3K27me3), thereby promoting selective gene silencing in normal stem cells and cancer cells. This epigenetic modification

\section{Biomed Central}

(c) 2015 Fornaro et al. Open Access This article is distributed under the terms of the Creative Commons Attribution 4.0 International License (http://creativecommons.org/licenses/by/4.0/), which permits unrestricted use, distribution, and reproduction in any medium, provided you give appropriate credit to the original author(s) and the source, provide a link to the Creative Commons license, and indicate if changes were made. The Creative Commons Public Domain Dedication waiver (http://creativecommons.org/publicdomain/zero/1.0/) applies to the data made available in this article, unless otherwise stated. 
is responsible for several key events in tumor development, including the occurrence of distant metastases and angiogenesis $[6,7]$. We have recently investigated the role of EZH2 single-nucleotide polymorphisms (SNPs) in mCRC patients treated with chemotherapy plus bevacizumab: the $\mathrm{C} / \mathrm{C}$ genotype for the rs3757441 SNP was associated with worse prognosis in terms of progression-free and overall survival [8]. This finding was subsequently confirmed in patients treated with chemotherapy alone, an evidence suggesting a prognostic significance of this SNP in mCRC [9]. We hypothesized that the $\mathrm{C} / \mathrm{C}$ genotype would create a transcription factor-binding site, thereby increasing the expression of the oncogenic EZH2 protein [8]. However, a definitive characterization of rs3757441 is still missing, since correlation between the SNP variants and EZH2/H3K27me3 protein expression in CRC samples has not been reported.

In the present study, we analyzed $E Z H 2$ rs3757441 genotype, EHZ2/H3K27me3 expression and pathological and molecular characteristics of 119 primary CRC samples.

\section{Methods}

\section{Sample selection and DNA isolation}

Consecutive CRC patients who underwent surgery on primary tumor and had their samples available for analyses at a single Institution (Unit of Pathology, Pisa) were identified. All patients provided written informed consent for sample collection and analysis. The study was conducted in accordance to the Declaration of Helsinki and the protocol was approved by the Ethics Committee of Pisa University Hospital. A total of 119 formalin-fixed paraffin-embedded tissues of primary CRCs were used for DNA extraction. DNA used for SNP analysis was extracted from normal colonic tissue, while tumor DNA was sequenced for KRAS and BRAF analyses. DNA was isolated using the QIAamp DNA Mini Kit (QIAGEN), following manufacturer's instructions. Concentration and purity of DNA were measured through a spectrophotometer.

\section{Genetic analyses}

Researchers who were not aware of EZH2 and H3K27 immunohistochemistry results and were blinded to patients' clinical data performed genetic analyses. EZH2 SNP [c.626-394C > T (rs3757441)] was analyzed through real-time PCR, as previously described [8]. KRAS exon 2 (codon 12-13) and BRAF exon 15 (codon 600) mutations were detected via real-time sequencing using PyroMark Gold Q96 reagents (QIAGEN) on PyroMarkTM Q96 ID instrument (Biotage, Sweden), as reported elsewhere [10]. Results were analyzed with PyroMark Q24 1.0.9 software.

\section{EZH2 and H3K27 immunohistochemistry}

Immunohistochemistry was performed on formalin fixed, paraffin-embedded tumor tissues, using previously validated protocols for both antibodies [11]. Involved pathologist carefully reviewed all CRC samples and selected tumor sections which were most representative of each tumor. Briefly, $5 \mu$ m-thick tissue sections were deparaffinized in xylene and rehydrated in a graded ethanol series. Slides were stained using a diaminobenzidine detection system preceded, only for EZH2, by heat-induced epitope retrieval involving immersion of tissue sections in a pre-warmed buffer solution (Target Retrieval Solution, DakoCytomation, Carpinteria, CA) and maintaining heat in a steamer at $988^{\circ} \mathrm{C}$ for $50 \mathrm{~min}$. To reduce nonspecific staining caused by endogenous biotin, the Endogenous Biotin Blocking Kit (Ventana Medical Systems, SA, Illkirch, Cedex, France) was employed, according to Manufacturer's instructions. EZH2 and H3K27me3 immunostaining was performed using monoclonal antibodies by Abcam (1:100 dilution and 1:50 dilution, respectively). Negative controls were obtained by omission of the primary antibodies.

Two pathologists were responsible for immunohistochemical evaluation and provided independent scoring results for both EZH2 and H3K27. In case of discrepancy, joint revision of the ambiguous samples was performed to assure the consistency of definitive results.

EZH2 expression was categorized as: $i$ ) percentage of positive cells (0-100 \%, evaluated as a continuous variable): the percentage of positive cells for each sample was determined as the average value of positive cells in 10 fields (approximately 100 cells per field were counted) at 10 High Power Field (HPF); ii) staining intensity, evaluated by a validated system [12]: we classified all cases in 4 categories [(-) less than $10 \%$ positive cells, any intensity; (1+) from $10-25 \%$, any intensity; (2+) from $25-75 \%$, any intensity; (3+) greater than $75 \%$, any intensity]; iii) Staining Index (SI), a semiquantitative method, evaluating both the heterogeneous distribution of positive cells and the differing intensity of the staining simultaneously: as described by Fluge et al. [13], SI is calculated as the product (0 to 9) of positive cells $(0,0 \% ; 1,<10 \% ; 2,10-50 \%$; and $3,>50 \%$ ) and staining intensity (negative to $3+$ ).

H3K27me3 expression was scored as follows: $i$ ) percentage of positive cells (0 - $100 \%)$; ii) staining intensity (as described for EZH2). In both cases, the pathologist performing immunohistochemistry analyses was blinded from genetic data.

\section{Statistical analysis}

EZH2 and H3K27 expression intensities were correlated with clinical and molecular features by means of chi-square test, T-test or ANOVA (as appropriate; significance was set at $P<0.05$ for two-tailed tests). 

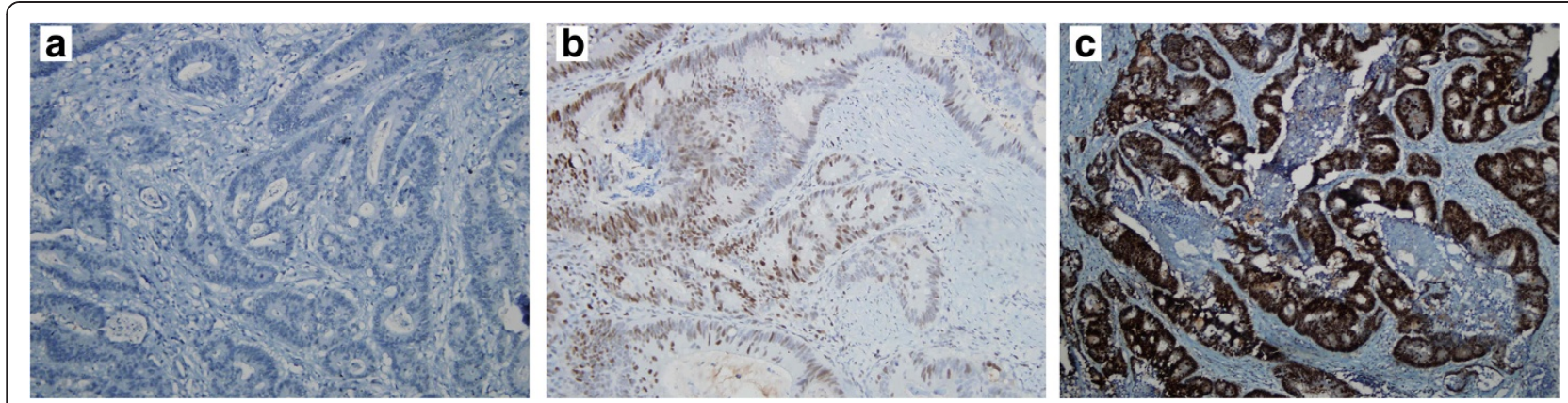

Fig. 1 Different EZH2 staining intensity at immunohistochemistry. EZH2 negative (panel a), EZH2 2+ (panel b) and EZH2 3+ (panel c) cases are shown. Negative: no staining; $1+$ (not shown): faint and barely perceptible staining; $2+$ : incomplete, weak to moderate staining; $3+$ : uniform, complete and intense staining

Neither pathologists performing immunohistochemistry nor molecular biologists performing genetic evaluation were directly involved in statistical analyses, which were conducted by independent investigators.

\section{Results}

\section{EZH2 and H3K27me3 expression}

Results of the expression analysis (Figs. 1,2) are summarized in Table 1. Prevalence of different SNP genotypes was in line with published data in Caucasian populations. Mean number of EZH2 or H3K27me3 positive cells was $46.2 \%$ (standard deviation, SD: 28.99) and $64.2 \%$ (SD: 29.10), respectively. KRAS-BRAF mutations occurred as expected from previous reports.

\section{Correlation of EZH2 rs3757441 variants with EZH2/ H3K27me3 expression}

Our primary goal was to investigate potential correlations between rs3757441 variants and EZH2/H3K27me3 expression. Moving from our previous reports [8, 9], we explored the association between protein expression and each genotype. Subsequently, we grouped the $\mathrm{C} / \mathrm{T}$ and $\mathrm{T} / \mathrm{T}$ genotypes (previously associated with better prognosis) and compared them with the $\mathrm{C} / \mathrm{C}$ genotype (Table 2 ).
We found significant differences among the three genotypes in terms of both EZH2 (3+ samples: $100 \% v s$. $39 \%$ vs. $48 \% ; P=0.028)$ and H3K27me3 (3+ samples: $100 \%$ vs. $35 \%$ vs. $40 \% ; P=0.022$ ) staining intensity. When the $\mathrm{C} / \mathrm{T}$ and $\mathrm{T} / \mathrm{T}$ cases were grouped, the samples harbouring the $\mathrm{C} / \mathrm{C}$ genotype showed significantly stronger EZH2 positivity compared to other genotypes (3+ samples: $100 \%$ vs. $44 \%$, respectively; $P=0.019$ ). The same significant association was reported for H3K27me3 (3+ samples among $\mathrm{C} / \mathrm{C}$ vs. C/T or $\mathrm{T} / \mathrm{T}$ : $100 \%$ vs. $38 \% ; P=0.009)$. Moreover, we observed a trend toward higher percentage of EZH2 positive cells $(60.0 \%$ vs. $46.0 \% ; P=0.288$ ) and higher SI (SI 4-9: $100 \%$ vs. $72 \%$; $P=0.322)$ in $\mathrm{C} / \mathrm{C}$ cases compared to $\mathrm{C} / \mathrm{T}$ or $\mathrm{T} / \mathrm{T}$ cases.

\section{Correlation of EZH2 rs3757441 variants with clinical and molecular characteristics}

The $\mathrm{C} / \mathrm{C}$ genotype was associated with higher tumor grade: all the 5 cases were scored as grade 3-4 tumors, while undifferentiated histology was reported in $37 \%$ and $38 \%$ of the $\mathrm{C} / \mathrm{T}$ and $\mathrm{T} / \mathrm{T}$ cases, respectively $(P=0.021)$ (Additional file 1: Table S1). We also observed a higher incidence of $B R A F$ mutation among $\mathrm{T} / \mathrm{T}$ cases $(16 \%$ vs. $0 \%$ and $2 \%$ among $\mathrm{C} / \mathrm{C}$ and $\mathrm{C} / \mathrm{T}$, respectively; $P=0.030)$.
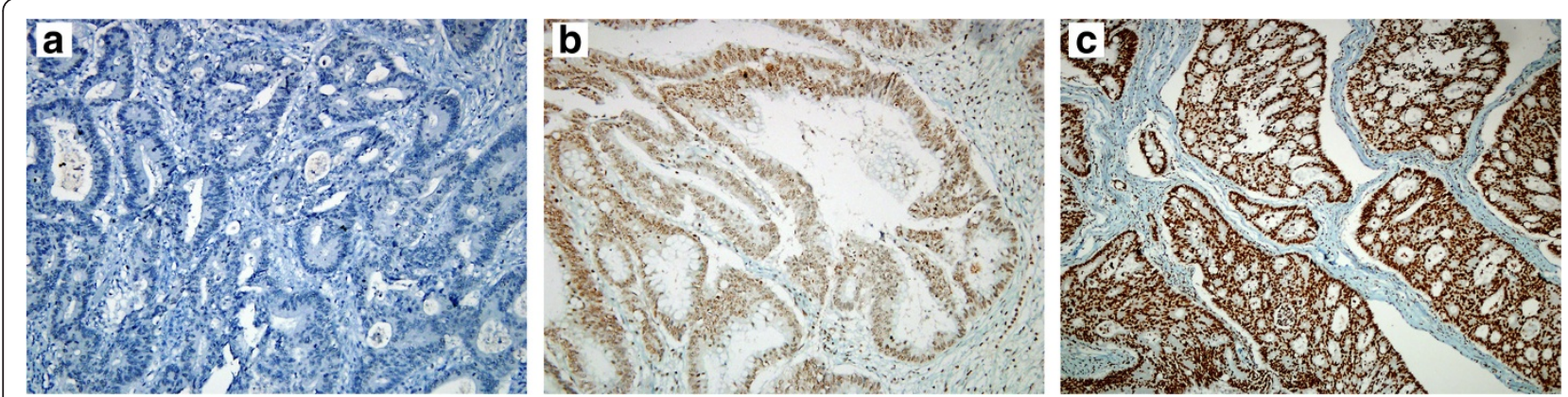

Fig. 2 Different H3K27me3 staining intensity at immunohistochemistry. H3K27me3 negative (panel a), H3K27me3 2+ (panel b) and H3K27me3 3+ (panel c) are shown. Negative: no staining; $1+$ (not shown): faint and barely perceptible staining; $2+$ : incomplete, weak to moderate staining; $3+$ : uniform, complete and intense staining 
Table 1 Specimen characteristics $(n=119)$

\begin{tabular}{|c|c|c|}
\hline Feature & Number & Percent \\
\hline \multicolumn{3}{|l|}{ Tumor stage } \\
\hline I & 5 & 4 \\
\hline$\|$ & 41 & 34 \\
\hline III & 52 & 44 \\
\hline IV & 21 & 18 \\
\hline \multicolumn{3}{|l|}{ Tumor grade } \\
\hline 2 & 76 & 64 \\
\hline $3-4$ & 43 & 36 \\
\hline \multicolumn{3}{|l|}{ Tumor site } \\
\hline right colon & 52 & 44 \\
\hline left colon & 45 & 38 \\
\hline rectum & 22 & 18 \\
\hline \multicolumn{3}{|c|}{ Mucinous histology } \\
\hline yes & 37 & 31 \\
\hline no & 82 & 69 \\
\hline \multicolumn{3}{|l|}{ KRAS status } \\
\hline wild-type & 67 & 56 \\
\hline mutant & 52 & 44 \\
\hline \multicolumn{3}{|l|}{ BRAF status } \\
\hline wild-type & 108 & 91 \\
\hline mutant & 11 & 9 \\
\hline \multicolumn{3}{|c|}{ EZH2 rs3757441 genotype } \\
\hline $\mathrm{C} / \mathrm{C}$ & 5 & 4 \\
\hline$C / T$ & 51 & 43 \\
\hline $\mathrm{T} / \mathrm{T}$ & 63 & 53 \\
\hline \multicolumn{3}{|c|}{ EZH2 staining intensity } \\
\hline $3+$ & 55 & 46 \\
\hline $2+$ & 33 & 28 \\
\hline $1+$ & 14 & 12 \\
\hline negative & 17 & 14 \\
\hline \multicolumn{3}{|c|}{ H3K27me3 staining intensity } \\
\hline $3+$ & 48 & 40 \\
\hline $2+$ & 40 & 34 \\
\hline $1+$ & 17 & 14 \\
\hline negative & 14 & 12 \\
\hline
\end{tabular}

Abbreviations: $n$, number; $\%$, percentage

Correlation of EZH2/H3K27me3 expression with clinical and molecular characteristics

We did not find any significant correlation between EZH2 expression and tumor stage, grading, histology and site of origin (Additional file 1: Table S2).

None of the investigated tumor features was associated with $\mathrm{H} 3 \mathrm{~K} 27 \mathrm{me} 3$ expression, with the exception of tumor site (H3K27me3 $3+$ cases were indeed more frequent among left-sided cancers compared with right- sided or rectal cancers: $55 \%$ vs. $33 \%$ vs. $27 \%$; $P=$ 0.028) (Additional file 1: Table S3). We also found a trend toward lower H3K27me3 expression in terms of percentage of positive cells $(67.9 \%$ vs. $57.7 \%$; $P=0.065)$ and staining intensity (3+ cases: $28 \%$ vs. $47 \%$; $P=$ 0.052 ) in poorly differentiated tumors.

$K R A S$ and $B R A F$ genotypes were not associated with EZH2 or H3K27me3 expression in our series.

Of note, we found a significant association between EZH2 and H3K27me3 staining intensity $(P=0.039)$. These results further corroborate the notion that higher EZH2 expression in CRC samples results in increased histone H3K27 methylation.

\section{Discussion}

We have already reported about the association between the $E Z H 2$ rs3757441 C/C genotype and worse prognosis in mCRC $[8,9]$ and showed that EZH2 mRNA expression (as measured in peripheral lymphocytes) was significantly higher among $\mathrm{C} / \mathrm{C}$ patients [8]. To characterize the role of this SNP in neoplastic cells, we analyzed EZH2 rs3757441 in normal colonic tissue (as the SNP accounts for germline variation in gene sequence) and evaluated EZH2 expression in tumor cells by immunohistochemistry in a series of 119 primary CRC. In this context, we found that the $\mathrm{C} / \mathrm{C}$ genotype was associated with significantly higher EZH2 expression (Table 2). Potential mechanisms by which the rs3757441 SNP may affect EZH2 expression have been proposed elsewhere [8].

Intriguingly, we also observed a strong association between the $\mathrm{C} / \mathrm{C}$ genotype and increased expression of $\mathrm{H} 3 \mathrm{~K} 27 \mathrm{me}$, which is the functional readout of EZH2 methylating activity. This result indicates that carriers of the $\mathrm{C} / \mathrm{C}$ genotype display higher EZH2 activity. In keeping with these findings, our data suggest that higher EZH2 expression results in increased EZH2 activation, as measured by $\mathrm{H} 3 \mathrm{~K} 27 \mathrm{me} 3$ expression, which is higher in EZH2 3+ cases.

Among the investigated clinico-pathological features, we observed a higher percentage of H3K27me3 cases among left colon tumors, compared with right colon or rectal cancers. This association was not significant for $\mathrm{EZH} 2$, even though EZH2 3+ samples were more frequent among left-sided tumors. It is known that rightand left-sided CRCs differ from biological and clinical perspectives $[14,15]$ : however, the small sample size of our series suggests caution in deriving any conclusion.

Finally, we investigated if $K R A S / B R A F$ mutations were associated with the EZH2 pathway, finding no significant correlation. Therefore, the suggested prognostic value of EZH2 expression and SNP appears to be independent of the KRAS and BRAF status: EZH2 genotyping may therefore add additional information to the conventional molecular profile of CRC cases. 
Table 2 Correlation of EZH2 rs3757441 variants with EZH2/H3K27me3 expression

\begin{tabular}{|c|c|c|c|c|c|c|c|c|c|c|}
\hline & $\mathrm{EZH} 2$ & & & & & & H3K27me3 & & & \\
\hline & $3+$ staining intensity & & $\%$ positive cells & & SI 4-9 & & $3+$ staining intensity & & $\%$ positive cells & \\
\hline Genotype (n) & $n(\%)$ & $P$ & mean (SD) & $P$ & $n(\%)$ & $P$ & $n(\%)$ & $P$ & mean (SD) & $P$ \\
\hline $\mathrm{C} / \mathrm{C}(5)$ & $5(100 \%)$ & & $60.0(15.8)$ & & $5(100 \%)$ & & $5(100 \%)$ & & $82.0 \%(4.472)$ & \\
\hline$C / T(51)$ & $20(39 \%)$ & & $46.0(27.7)$ & & $38(74 \%)$ & & $18(35 \%)$ & & $60.0 \%(30.53)$ & \\
\hline $\mathrm{T} / \mathrm{T}(63)$ & $30(48 \%)$ & 0.028 & $46.0(30.3)$ & 0.571 & 44 (70\%) & 0.328 & 25 (40\%) & 0.022 & $66.19 \%(28.54)$ & 0.200 \\
\hline C/C (5) & $5(100 \%)$ & & $60.0 \%(15.8)$ & & $5(100 \%)$ & & $5(100 \%)$ & & $82.0 \%(4.472)$ & \\
\hline $\mathrm{C} / \mathrm{T}$ or $\mathrm{T} / \mathrm{T}(114)$ & $50(44 \%)$ & 0.019 & $46.0 \%(29.1)$ & 0.288 & $82(72 \%)$ & 0.322 & $43(38 \%)$ & 0.009 & $63.42 \%(29.47)$ & 0.163 \\
\hline
\end{tabular}

Abbreviations: \%, percentage; $n$, number; $P, P$-value; $S D$, standard deviation; $S I$, Staining Index

In the present series, we did not investigate the association between the EZH2 SNP or the EZH2/H3K27me3 expression and survival outcome: patient heterogeneity in terms of disease stage, adjuvant treatments and length of follow up might have affected the results, leading to inconclusive or confounding findings.

Our study has several limitations that should be taken into account in the interpretation of the results. First, we retrieved only 5 patients with the $\mathrm{C} / \mathrm{C}$ genotype for the EZH2 rs3757441 SNP: therefore, our findings cannot be considered definitive and further analyses should be conducted in larger patient cohorts to confirm the association between EZH2 SNP variants and protein expression. Notably, the results described in the present manuscript are in line with our previous report about mRNA analysis on peripheral lymphocytes from CRC patients [8]. This investigation revealed that EZH2 mRNA expression is higher in homozygous $\mathrm{C}$ allele carriers, with no difference between $\mathrm{C} / \mathrm{T}$ and $\mathrm{T} / \mathrm{T}$ subjects [8]. In our opinion, these two investigations support further evaluation of this SNP in CRC and possibly in other malignancies. Indeed, other researchers have recently suggested the relevance of the rs3757441 EZH2 SNP in hepatocellular carcinoma: Yu et al. reported that patients who carried at least one $C$ allele have a higher lymphnode-metastasis risk than did patients carrying the wildtype allele [16], thus confirming the potential functional relevance of this SNP in cancer cells. Secondly, a standardized score to evaluate EZH2 (and H3K27me3) positivity by immunohistochemistry is lacking: future studies should thus clarify which is the preferred method to assess this parameter (i.e. the percentage of positive cells, the intensity of staining or a combination of both, as for the SI proposed by other authors [13]).

\section{Conclusions}

To conclude, we characterized the EZH2 rs3757441 SNP, showing that the $\mathrm{C} / \mathrm{C}$ variant is associated with higher EZH2 expression and activity in the neoplastic tissue. This SNP genotype has been linked with worse prognosis among mCRC patients treated with chemotherapy with or without an anti-vascular endothelial growth factor antibody: our data confirm in primary tumors that the $\mathrm{C}$ allele is linked with increased EZH2 expression and activity, thus providing additional evidence for a functional relevance of this SNP in CRC biology. As anti-EZH2 drugs are under development and early clinical evaluation [17], this SNP may serve as a potential predictive biomarker for such agents in CRC patients.

\section{Additional file}

Additional file 1: Table S1: Correlation of EZH2 rs3757441 variants with clinical and molecular characteristics. Abbreviations: \%, percentage; $n$, number; $P, P$-value. Table S2: EZH2 expression according to tumour characteristics. Abbreviations: \%, percentage; $n$, number; $P, P$-value; SD, standard deviation; SI, Staining Index. Table S3: H3K27me3 expression according to tumour characteristics. Abbreviations: \%, percentage; $n$, number; $P$, $P$-value; $S D$, standard deviation. (PDF $98 \mathrm{~kb}$ )

\section{Abbreviations}

CRC: colorectal cancer; H3K27me3: histone H3 trimethylation on lysine 27; mCRC: metastatic colorectal cancer; PcGs: Polycomb Group genes; PCR: polymerase chain reaction; PRCs: Polycomb Repressive Complexes; SNPs: single-nucleotide polymorphisms.

\section{Competing interests}

The authors declare that they have no competing interests.

\section{Authors' contributions}

$L F, C V$ and FC designed the study, performed statistical analyses and drafted the manuscript. PF and GF performed immunohistochemistry. VDG, EP, ES, $C L, G F$ and $F C$ performed genetic experiments. $L F, P F, C V, G M, F L, G F, ~ Y W$, $\mathrm{RD}, \mathrm{AF}$ and FC supervised the data. All authors read and approved the final manuscript.

\section{Acknowledgements}

The work was supported by funds from Fondazione ARCO Onlus (Azioni Ricerche e Cure in Oncologia - Pisa, Italy).

\section{Author details}

'Unit of Medical Oncology 2, Azienda Ospedaliero-Universitaria Pisana, Istituto Toscano Tumori, Pisa, Italy. ${ }^{2}$ Unit of Pathology, Department of Surgical, Medical and Molecular Pathology and Critical Care, University of Pisa, Pisa, Italy. ${ }^{3}$ Department of Biology, Unit of Genetics, University of Pisa, Pisa, Italy. ${ }^{4}$ Experimental Therapeutics, BCCA Cancer Research Centre, Vancouver, BC, Canada. ${ }^{5}$ Vancouver Prostate Centre, Vancouver, BC, Canada. ${ }^{6}$ Pharmacology Unit, Department of Clinical and Experimental Medicine, University of Pisa, Pisa, Italy. ${ }^{7}$ Department of Life Health and Chemical Sciences, The Open Universit, Milton Keynes, UK. 
Received: 15 June 2015 Accepted: 30 October 2015

Published online: 09 November 2015

\section{References}

1. Siegel R, Naishadham D, Jemal A. Cancer statistics, 2013. CA Cancer J Clin. 2013:63:11-30.

2. Douillard JY, Oliner KS, Siena S, Tabernero J, Burkes R, Barugel M, et al. Panitumumab-FOLFOX4 treatment and RAS mutations in colorectal cancer. New Engl J Med. 2013;369:1023-34.

3. Souglakos J, Philips J, Wang R, Marwah S, Silver M, Tzardi M, et al. Prognostic and predictive value of common mutations for treatment response and survival in patients with metastatic colorectal cancer. $\mathrm{Br} \mathrm{J}$ Cancer. 2009;101:465-72.

4. You JS, Jones PA. Cancer genetics and epigenetics: two sides of the same coin? Cancer Cell. 2012;22:9-20

5. Simon JA, Lange CA. Roles of the EZH2 histone methyltransferase in cancer epigenetics. Mutat Res. 2008;647:21-9.

6. Crea F, Paolicchi E, Marquez VE, Danesi R. Polycomb genes and cancer: time for clinical application? Crit Rev Oncol Hematol. 2012;83:184-93.

7. Crea F, Fornaro L, Bocci G, Sun L, Farrar WL, Falcone A, et al. EZH2 inhibition: targeting the crossroad of tumor invasion and angiogenesis. Cancer Met Rev. 2012;31:753-61.

8. Crea F, Fornaro L, Paolicchi E, Masi G, Frumento P, Loupakis F, et al. An $\mathrm{EZH} 2$ polymorphism is associated with clinical outcome in metastatic colorectal cancer patients. Ann Oncol. 2012;23:1207-13.

9. Fornaro L, Crea F, Masi G, Paolicchi E, Loupakis F, Graziano F, et al. EZH2 polymorphism and benefit from bevacizumab in colorectal cancer: another piece to the puzzle. Ann Oncol. 2012;23:1370-1.

10. Loupakis F, Cremolini C, Fioravanti A, Orlandi P, Salvatore L, Masi G, et al. EGFR ligands as pharmacodynamic biomarkers in metastatic colorectal cancer patients treated with cetuximab and irinotecan. Target Oncol. 2014;9:205-14.

11. Benard A, Goossens-Beumer IJ, van Hoesel AQ, Horati H, Putter H, Zeestraten EC, et al. Prognostic value of Polycomb proteins EZH2, BMI1 and SUZ12 and histone modification H3K27me3 in colorectal cancer. PLoS One. 2014;9(9), e108265

12. Kleer CG, Cao Q, Varambally S, Shen R, Ota I, Tomlins SA, et al. EZH2 is a marker of aggressive breast cancer and promotes neoplastic transformation of breast epithelial cells. Proc Natl Acad Sci U S A. 2003;100:11606-11.

13. Fluge $\varnothing$, Gravdal K, Carlsen E, Vonen B, Kjellevold K, Norwegian Gastrointestinal Cancer Group, et al. Expression of EZH2 and Ki-67 in colorectal cancer and associations with treatment response and prognosis. Br J Cancer. 2009;101:1282-9.

14. Loupakis F, Yang D, Yau L, Feng S, Cremolini C, Zhang W, et al. Primary tumor location as a prognostic factor in metastatic colorectal cancer. J Natl Cancer Inst. 2015; 107. doi: 10.1093/jnci/dju427

15. Weiss JM, Pfau PR, O'Connor ES, King J, LoConte N, Kennedy G, et al. Mortality by stage for right- versus left-sided colon cancer: analysis of surveillance, epidemiology, and end results-Medicare data. J Clin Oncol. 2011;29:4401-9.

16. Yu YL, Su KJ, Hsieh YH, Lee HL, Chen TY, Hsiao PC, et al. Effects of EZH2 polymorphisms on susceptibility to and pathological development of hepatocellular carcinoma. PLoS One. 2013;8(9), e74870.

17. Fillmore CM, Xu C, Desai PT, Berry JM, Rowbotham SP, Lin YJ, et al. EZH2 inhibition sensitizes BRG1 and EGFR mutant lung tumours to Topoll inhibitors. Nature. 2015;520:239-42.

\section{Submit your next manuscript to BioMed Central and take full advantage of:}

- Convenient online submission

- Thorough peer review

- No space constraints or color figure charges

- Immediate publication on acceptance

- Inclusion in PubMed, CAS, Scopus and Google Scholar

- Research which is freely available for redistribution 